\title{
A study of HIV/AIDS related knowledge, attitude and behaviors among female sex workers in Shanghai China
}

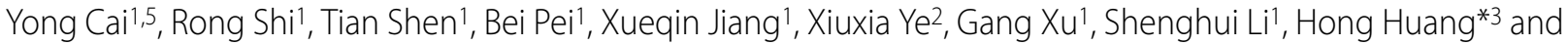 \\ Meili Shang 4
}

\begin{abstract}
Background: China is currently facing a rapid and widespread increase in human immunodeficiency virus (HIV)/ acquired immunodeficiency syndrome (AIDS). The activities of female sex workers (FSWs) have contributed to the mounting epidemic of HIV/AIDS and other sexually transmitted diseases (STDs). Therefore, this study aimed to assess the HIV/AIDS-related knowledge, attitude and risk behaviors among FSWs operating in Shanghai China.

Methods: A cross-sectional study was conducted in five districts of Shanghai, including three suburbs and two downtown locales. We adopted a cluster randomized sampling method to obtain ten geographic sites which consisted of one or more communities/villages proximal to a location where FSWs were accessible. A total of 324 FSWs from 109 Xitou Fang, massage parlors and hair salons who explicitly provided sexual services were enrolled in the study. Each participant completed a questionnaire survey and interview aimed to collect information on the individual's knowledge, attitude, and behaviors associated with risk for HIV/AIDs.

Results: The overall correct answer rate of HIV/AIDS-related knowledge was $60.8 \%$, and the knowledge of FSWs from downtown areas was significantly higher than those from suburban areas $(P<0.05)$. The percentage of FSWs who reported having experiences in commercial sexual services without the use of condoms was $33.6 \%$. Condom slippage or breakage was reported as having occurred at least once by 51.2\% of the FSWs. FSWs from suburban areas were found to more often engage in high-risk behaviors, including oral and anal sex, than those from downtown areas $(P<$ 0.001). Many of the FSWs (65.7\%) reported having non-client sexual partners (most were identified as boyfriends or husbands); however, condom usage with these partners were lower (34.3\%).

Conclusions: Based on the findings from our survey, we advise that promotion of HIV/AIDS-related knowledge be targeted towards FSWs in Shanghai, especially those operating in the suburbs. HIV prevention efforts, such as urging constant condom usage with both clients and steady partners, should be sustained and reinforced among the female sex workers population.
\end{abstract}

\section{Background}

The HIV/AIDS epidemic is one of the world's most serious public health and social problems. In the past decade China has experienced a rapid increase in HIV/AIDS cases; the number of Chinese people living with HIV has continued to raise despite the availability of effective prevention strategies [1]. At the end of October 2009, the

* Correspondence: huanghong096@hotmail.com

3 Xinhua Hospital affiliated with Shanghai Jiaotong University School of Medicine, (No.1665, Kongjiang Road), Shanghai, (200092), PR China Full list of author information is available at the end of the article cumulative reported HIV-infected patients, including those who had progressed to AIDS, stood at 319877. More alarming statistics came from the Chinese government, who along with the World Health Organization (WHO) and the United Nations Program on HIV/AIDS (UNAIDS) estimated that closer to 740000 people are infected with HIV in China, including 105000 individuals suffering from AIDS. This past year, new cases of HIV infection in China numbered at about 48,000 [2].

Four major factors have been identified as significant contributors to the HIV/AIDs epidemic among the gen- 
eral population of China. The first is drug abuse; intravenous drug use and needle sharing is a well-established risk factor for the spread of HIV/AIDS. Moreover, the practice of illicit drug usage lends to uninhibited and uncontrolled behaviors conducive to HIV transmission through sexual contact. The second factor is the so-called 'floating population'- the approximately 150 million migrant workers in China. As much as $73 \%$ of migrants originate from poorer regions of the country and come to work in the cities as laborers, restaurant workers and sex workers. This portion of the population have been considered as the 'tipping point' for the current HIV/STDs epidemic [3,4]. It was suggested that rural-to-urban migration may play a crucial role in shifting the HIV/STI epidemic by broadening social and sexual mixing $[5,6]$. The third factor is unprotected and high-risk sexual activity, including that by sex workers, the male homosexual population and individuals having multiple sex partners. The fourth factor is the lack of knowledge about HIV/AIDS, which can be subdivided into two components: the lack of simple knowledge of HIV/AIDS and discriminatory attitude which will induces anti-social behavior.

Heterosexual contact is now considered the most common mode of transmission of HIV infection in China. The latest assessment report indicates that heterosexual transmission accounted for $42.2 \%$ of China's newlyinfected cases of HIV diagnosed in 2009. In Shanghai, 886 new HIV infections were reported in 2009; all patients were under the age of 45 and $63.7 \%$ of them were infected through a sexual transmission route. Female sex workers are considered a significant contributor to the heterosexual transmission rates of HIV since their unprotected anonymized sexual activities act as a "bridge" to spread HIV/AIDs to the general population.

The commercial sex trade has a long and complex history in China, as has been greatly influenced by the political and economic changes experienced by the Chinese republic during the 20th century $[7,8]$. During the past 25 years, China's open door policy and economic reforms have been accompanied by a remarkable resurgence in the commercial sex sector. Indeed, the Chinese National Sentinel Surveillance System concluded that sexual transmission was the most common route of new HIV infections in 2007 to 2009. Female sex workers represent an important reservoir of sexually transmitted diseases (STDs), including HIV. For example, studies have shown that $20 \%$ to $48.8 \%$ of the female sex workers examined were carrying more than one kind of STD, the most common of which was gonorrhea. However, the rates of HIV were found to be generally lower than for the other types of STDs detected. In China, providing commercial sexual services is illegal and most sex workers operate in karaoke bars, massage parlors, saunas, hair and beauty salons, while some solicit clients from the street or in parks [9]. In many cases, these female sex workers are simply unaware of the risk of HIV/AIDS, since most of them are young girls with limited education who have migrated from poor rural areas to towns or cities [10]. The prevalence of STDs and HIV in FSWs suggests a critical need for prevention efforts and health education. Here, we conducted a cross-sectional study to evaluate the HIV/ AIDS-related knowledge, attitude and behaviors among female sex workers in Shanghai. The information gathered from this study will contribute to future construction and institution of effective strategies towards HIV/ AIDS prevention among this particular population.

\section{Methods}

The objectives of the study were to document the sociodemographic and sex work characteristics of FSWs in Shanghai in order to assess the HIV/AIDS-related knowledge, attitude and risk behaviors of this population. This study was approved by the Shanghai Medical Ethics Committee and the Shanghai Municipal Health Bureau, and all participants were informed of the study's objectives and provided consent.

\section{Study area}

Shanghai is one of the largest metropolitan areas in China where the HIV/AIDS transmission route is largely attributed to heterosexual contact. Since provision of commercial sexual services is illegal in China and most sex workers generally operate covertly, it is impossible to estimate the actual number of FSWs operating in Shanghai. Non-governmental organizations and community hospitals are the best links to the FSW population, and we relied on their knowledge to conduct our study among five districts (Songjiang, Putuo, Qingpu, Hongkou and Pudong) of Shanghai where access to FSWs was considered feasible.

\section{Sample and Procedure}

We adopted a cluster sampling method in Shanghai to obtain ten random geographic sites from the five districts (including two downtown areas and three suburbs). Each geographic site consisted of one or more locations (community/villages) proximal to where FSWs were accessible; in particular, accessible FSWs were defined as operating in hotspots such as Xitou Fang, massage parlors and hair salons. We performed site visits to each of the hotspots where commercial sexual services were provided. We noted that only 2-4 female sex workers operated out of each hotspot.

By carrying out a detailed review of the literature and performing a small-scale pre-survey with a limited portion of the female sex workers in Shanghai, we found the risk behaviors, such as unprotected sexual intercourse, 
was $38 \%$. By taking a cross-sectional sample size estimation approach, the error was calculated as $\mathrm{d}=0.15 \mathrm{P}, \mathrm{P}=$ $38 \%, \mathrm{Q}=1-\mathrm{P}=62 \%, \alpha=0.05$. The sample size calculation method used was: $\mathrm{n}=\mathrm{t} 2 \mathrm{PQ} / \mathrm{d} 2=250$. The intra-cluster correlation coefficient (ICC) of the primary outcome is known to play a key role in the design and analysis of cluster randomized trials, in which clusters such as health care organizations, school classes, or geographic areas are randomized to trial arms and outcomes are measured for individuals within those clusters [11]. The ICC for condom use ranged from 0.01-0.08 in other studies [12,13]. Since it is very difficult to obtain a sufficiently large sample population of female sex workers in China, we used ICC $=0.01$ to adjust the sample size.

$$
N_{\text {adjucted }}=n \times[1+\operatorname{ICC}(m-1)]=250 \times[1+0.01(25-1)]=310
$$

Our final sample population was made up of 324 female sex workers from Shanghai districts' hotpots located in ten geographic sites. Individuals in this cohort completed the entire questionnaires administered in-person. Indepth interviews were also conducted with female sex workers, in order to collect detailed information about condom usage and other specific behaviors related to HIV/AIDS. Prior to participation, we explained the study purpose to each of the FSWIs and emphasized that participation was voluntary and anonymous.

\section{Measures}

The interviewer-administered questionnaire of HIV/ AIDS was designed to collect data on individual's socioeconomic and demographic characteristics, including participant's hometown, age, marital status, education levels and incomes. A total of 24 items addressed HIV/ AIDS-related knowledge, including basic information on HIV, transmission, and self-protection; another 20 items focused on sources of HIV/AIDS-related information, such as risky sexual practices, drug use, and condom use. To assess the participant's knowledge on HIV/AIDS, participants were asked to respond to the 24 knowledge items with 'yes', 'no' or 'do not know'. Correct answers were credited with a score of one, while incorrect answers or responses of "do not know" received a score of zero. The sum of each question's score was converted into a total score, with the maximum being 24 . Overall correct HIV/STDs knowledge was calculated by using the average score divided by the maximum score.

\section{Statistical Analysis}

Data were double-entered using Epidata3.0 software. All statistical analyses were performed using the Statistical Package for Social Sciences (SPSS) for Windows. Generalized assessments were made using mean, standard deviation and percentages. Chi-square test was used to compare differences of socio-demographic characteristics between suburb and city central. Chi-square test was also used to compare differences in HIV/AIDS-related knowledge between suburbs and downtown areas. We estimated the ICC as 0.01 and computed adjustments $X^{2}{ }_{r s}$ to the Pearson chi-square, as proposed by Rao and Scott [14], to adjust for the clustering design effect. Statistical significance was considered at $P<0.05$ (two-tailed).

\section{Results}

Socio-demographic characteristics of study participants

Study participants included 324 FSWs from three suburbs and two downtown areas in Shanghai. All participants had migrated from poor rural areas of western China, and most had come from either Sichuan, Hunan, or Hubei. The mean age of the sample population was 25.2 years $(\mathrm{SD}=4.9$; range, $15.5-37.1$ years) and $53.1 \%$ of the FSWs were under 25. About half of FSWs were never married and most had received limited education (78.1\% stopped schooling before high school). The proportion of monthly income was found to be significantly higher among FSWs from downtown areas $(P<0.05)$. However, there was no significant difference found between suburbs and downtowns in age, marital status or education level $(P>0.05)$ (Table 1$)$.

\section{HIV/AIDS-related knowledge among FSWs}

The comprehensive correct rate of HIV/STD knowledge was $60.8 \%$. The correct rate for FSWs from downtown areas was significantly higher than that for FSWs from the suburbs $(P<0.05)$ (Table 2$)$.

\section{HIV/AIDS-related attitudes among FSWs}

More than $50 \%$ of the FSWs indicated that they believed HIV-infected individuals should be forcibly isolated. But, more than $80 \%$ thought that it was reasonable to help an HIV-infected individual.

\section{HIV/AIDS-related behaviors among FSWs}

The average age of sexual debut was 18.80 years among the 324 FSW study participants. The average age when these women started selling sex was 22.84 years, and women reported working as sex workers for a average of 2.93 years. There were no significantly differences found between suburbs and downtowns for these variables $(P>$ 0.05).

In our interviews, most FSWs regarded prostitution as an experience of life and her own choice, but warned themselves not to be "too erotic" (that was, providing different kinds of sexual behaviors besides traditional intercourse). Three-hundred-and-seventeen of the 324 participants (97.8\%) provided vaginal sex, and 141 of them $(43.5 \%)$ provided oral sex. Only a small portion $(\mathrm{n}=$ $57 ; 17.6 \%)$ reported ever having had anal sex with clients. 
Table 1: Socio-demographic characteristics of FSWs from downtown and suburban areas

\begin{tabular}{|c|c|c|c|c|c|c|c|c|}
\hline \multirow[t]{2}{*}{ Characteristics } & \multicolumn{2}{|c|}{ Downtown $(n=158)$} & \multicolumn{2}{|c|}{ Suburban $(n=166)$} & \multicolumn{2}{|c|}{ Total $(n=324)$} & \multirow[t]{2}{*}{$X_{r s}^{2}{ }^{a}$} & \multirow[t]{2}{*}{$P$-value } \\
\hline & $n$ & $\%$ & $n$ & $\%$ & $n$ & $\%$ & & \\
\hline \multicolumn{9}{|l|}{ Age: } \\
\hline mean $\pm S D$ (range) & \multicolumn{2}{|c|}{$25.3 \pm 4.8(15.5-36.2)$} & \multicolumn{2}{|c|}{$25.9 \pm 5.1(16.5-37.1)$} & \multicolumn{2}{|c|}{$25.5 \pm 4.9(15.5-37.1)$} & & \\
\hline$<25$ & 88 & 55.7 & 84 & 50.6 & 172 & 53.1 & 0.437 & 0.748 \\
\hline $25+$ & 70 & 44.3 & 82 & 49.4 & 152 & 46.9 & & \\
\hline \multicolumn{9}{|l|}{ Marital status } \\
\hline Never married & 76 & 48.1 & 70 & 42.2 & 146 & 45.1 & 0.751 & 0.483 \\
\hline Married or divorced & 82 & 51.9 & 96 & 57.8 & 178 & 54.9 & & \\
\hline \multicolumn{9}{|l|}{ Education } \\
\hline$<=$ Middle school & 119 & 75.3 & 134 & 81.7 & 224 & 78.1 & 1.076 & 0.184 \\
\hline High school + & 39 & 24.7 & 32 & 19.3 & 71 & 21.9 & & \\
\hline \multicolumn{9}{|l|}{ Monthly income (RMB) } \\
\hline$<2500$ Yuan & 96 & 60.8 & 122 & 73.5 & 218 & 67.3 & 14.432 & $<0.050$ \\
\hline $2500+$ Yuan & 62 & 39.2 & 44 & 26.5 & 106 & 32.7 & & \\
\hline
\end{tabular}

a: We estimated the ICC as 0.01 . Computed adjustments $X_{r s}^{2}$ to the Pearson chi-square were made as proposed by Rao and Scott [12] that adjusted for the clustering design effect (10 geographic sites).

$70.1 \%$ of the FSWs always used condoms to protect themselves when they had vaginal sex with clients, and this figure was $57.9 \%$ for anal sex but only $22.7 \%$ for oral sex. $75.3 \%$ of women reported having used condoms in their most recent sexual intercourse. 51.2\% had the experience of condom breakage or slippage during sex. $33.6 \%$ of FSWs had ever had sex without a condom because clients paid more money and looked clean. Many women (65.7\%) had non-client sexual partners (most were boy- friends or husbands); however, condom usage with these partners was lower (34.3\%) (Table 3).

\section{Discussion}

Female sex workers lacking self-protection consciousness are at an extremely high risk of acquiring HIV-1 (HIV). This vulnerable group is uniquely poised to amplify HIV transmission to the general population. Prostitution has been a visible part of Chinese culture throughout history,

Table 2: HIV/STDS-related knowledge of FSWs from downtown and suburban areas

\begin{tabular}{|c|c|c|c|}
\hline \multirow[t]{2}{*}{ Questionnaire items } & \multicolumn{3}{|c|}{ Correct rate (\%) } \\
\hline & total & suburban & downtown \\
\hline Overall correct answers of HIV/STDs knowledge items & 60.8 & 53.3 & $68.6 \mathrm{~b}$ \\
\hline Infection with HIV can have no symptoms & 35.2 & 24.1 & $46.8 \mathrm{~b}$ \\
\hline Mosquito bites can spread HIV & 41.4 & 37.3 & 45.6 \\
\hline the use of condoms can reduce the risk of HIV infection & 88.9 & 84.3 & $93.7 \mathrm{~b}$ \\
\hline HIV can be spread by sharing tableware & 69.8 & 56.5 & $83.5 \mathrm{~b}$ \\
\hline HIV can be spread by sharing toilet or bathtub & 52.5 & 34.9 & $60.8 \mathrm{~b}$ \\
\hline HIV infected blood is a risk factor for HIV transmission & 89.5 & 86.7 & 92.4 \\
\hline Unprotected sexual relations is a risk factor for HIV infection & 82.1 & 72.3 & $92.4 \mathrm{~b}$ \\
\hline Unprotected oral sex may transmit HIV & 51.2 & 48.2 & 54.4 \\
\hline
\end{tabular}

$\mathrm{b}: \mathrm{P}<0.05$ by using $X^{2}{ }_{r s}$ to adjust the Pearson chi-square 
Table 3: Sexual behaviors and condom use among 324 FSWs

\begin{tabular}{|c|c|c|}
\hline Questionnaire items & $n$ & $\%$ \\
\hline \multicolumn{3}{|l|}{ Condom use during vaginal sex } \\
\hline Always & 227 & 70.1 \\
\hline Sometimes & 89 & 27.4 \\
\hline Never & 8 & 2.5 \\
\hline Total & 324 & 100.0 \\
\hline \multicolumn{3}{|l|}{ Condom use during oral sex } \\
\hline Always & 32 & 22.7 \\
\hline Sometimes & 69 & 48.9 \\
\hline Never & 40 & 28.3 \\
\hline Total & 141 & 100.0 \\
\hline \multicolumn{3}{|l|}{ Condom use during anal sex } \\
\hline Always & 33 & 57.9 \\
\hline Sometimes & 18 & 31.6 \\
\hline Never & 6 & 10.5 \\
\hline Total & 57 & 100.0 \\
\hline Condom used in most recent sexual intercourse with a client & 244 & 75.3 \\
\hline \multicolumn{3}{|l|}{ Commercial clients per day } \\
\hline$<=1$ & 133 & 41.0 \\
\hline 2 & 114 & 35.2 \\
\hline$>=3$ & 77 & 23.8 \\
\hline \multicolumn{3}{|l|}{ In most recent three episodes of sexual intercourse, times condoms use } \\
\hline 0 & 7 & 2.2 \\
\hline 1 & 49 & 15.1 \\
\hline 2 & 66 & 20.4 \\
\hline 3 & 198 & 61.1 \\
\hline Had the experience of condom breakage or slippage during sex & 166 & 51.2 \\
\hline \multicolumn{3}{|l|}{ Had sex without a condom because clients paid more money and looked clean } \\
\hline Yes & 109 & 33.6 \\
\hline No & 215 & 66.4 \\
\hline Had sex with non-client sexual partners & 213 & 65.7 \\
\hline Steady lover/boyfriend/husband & 132 & 62.1 \\
\hline Known friend & 60 & 28.2 \\
\hline others & 21 & 9.7 \\
\hline Used a condom with non-client partner in most recent sexual intercourse & 73 & 34.3 \\
\hline \multicolumn{3}{|l|}{ Reasons for using a condom? } \\
\hline Men are dirty & 133 & 41.1 \\
\hline Worried about pregnancy & 176 & 54.3 \\
\hline Worried about STDs including HIV & 261 & 80.6 \\
\hline \multicolumn{3}{|l|}{ Usually, where did you obtain your condoms? } \\
\hline Family planning & 51 & 15.7 \\
\hline Purchased at pharmacy & 243 & 75.0 \\
\hline Owner provided & 30 & 9.3 \\
\hline
\end{tabular}


but the devastating health consequences of the commercial sex trade were not documented until the 20th century. Thus, we conducted our research among female sex workers of Shanghai to access the baseline of HIV/AIDSrelated knowledge and risk behaviors.

As shown by research of an Indian population of FSWs [15], we also found that negotiating condom use with regular partners (usually husbands or boyfriends) was difficult and more complex than with occasional clients in Shanghai. In our research, all FSWs worked outside their home towns and a significant proportion (65.7\%\%) had regular sex partners who were not paying clients but defined as husbands or boyfriends. In-depth interviews revealed that FSWs usually disguised their sources of income to their husbands or boyfriends, so their male partners may implicitly trust that their wives/girlfriends are not having unprotected sex with anyone else, making it difficult for the sex workers to initiate condom usage with them. In general, condom use among FSWs is low with steady partners, but higher with clients, especially new clients [16]. It appears that although sex workers are able to be convinced to use condoms with clients, it was more difficult for them to initiate condom usage with non-paying partners.

In our research, the consistent condom usage with clients among female sex workers was reported as being $70.1 \%$ during vaginal sex, a higher percentage than was reported several years ago in China [17-20] which then only ranged from $17 \%$ to $50 \%$. However, we found that $33.6 \%$ of the FSWs had had sex without condoms when clients paid more money and looked clean. They believed that clients who looked well-educated would be gentle and clean, which would reduce the risk of HIV and other STDs. In addition, the unprotected sexual behaviors during anal sex and oral sex were still reported as high, as is shown in Table 3. These findings served as a reminder that it is urgently necessary to reinforce the notion of selfprotection during sexual encounters among FSWs since HIV/STDs could also be transmitted by unprotected anal sex or oral sex. Successful prevention strategies already established have included peer education and counseling to reduce high-risk sexual behavior by reducing client numbers and increasing use of male condoms. This research presented herein will aid in future studies on intervention techniques among FSWs of Shanghai in the future since interventions targeted at FSWs were among the most cost-effective public health strategies available to curb HIV transmission.

\section{Conclusions}

We conducted a study on a medium-sized sample of female sex workers in Shanghai, a subgroup in China with potentially greater risk for HIV/STI. Our study not only examined the current knowledge of HIV/AIDs among this group, but also identified the risky sexual behaviors practiced. HIV prevention efforts, such as promoting constant condom usage with clients and steady partners, among female sex workers should be sustained and reinforced. The results of our analysis have provided data to promote interventions for improving commercial sex behavior and the sexual health of FSWs in the future. In addition, the comparative study between FSWs from downtown and suburb areas indicated that more attention needs to be paid to HIV/AIDS prevention among FSWs operating in suburbs.

\section{Competing interests}

The authors declare that they have no competing interests.

\section{Authors' contributions}

All authors contributed the design of this research. CY drafted the manuscript and has been involved in the interpretation of the data. GX and RS performed statistical analyses; TS, BP, XJ, XY, SL and MS played a major role in the field survey. $\mathrm{HH}$ made a substantial contribution to the interpretation of the data and has been involved in revision of the manuscript through all stages. All authors read and approved the final manuscript.

\section{Acknowledgements}

This research was funded by the Shanghai Municipal Health Bureau (project number: 08GWQ055), general program of humanities and social studies, China Ministry of Education and Shanghai Education Committee (Project of Innovation). Our sincere gratitude is due to the study participants who made this research possible.

\section{Author Details}

1School of Public Health affiliated with Shanghai Jiaotong University School of Medicine, (No.227, South Chongqing Road), Shanghai, (200025), PR China, 2Shanghai Children's Medical Center, affiliated with Shanghai Jiaotong University School of Medicine, (No.1678, Dongfang Road), Shanghai, (200127), PR China, ${ }^{3}$ Xinhua Hospital affiliated with Shanghai Jiaotong University School of Medicine, (No.1665, Kongjiang Road), Shanghai, (200092), PR China, ${ }^{4}$ Community Sanitary Service Center of North Sichuan Road, Hongkou Distinct, (No. 300, Tanggu Road), Shanghai, (200085), PR China and ${ }^{5}$ School of Public Health affiliated with Shanghai Fudan University, (No.130, Dong'an Road), Shanghai,(200032), PR China

Received: 15 December 2009 Accepted: 28 June 2010 Published: 28 June 2010

\section{References}

1. Dandona Rakhi, Dandona Lalit, Gutierrez Juan Pablo, Kumar Anil G McPherson Sam, Samuels Fiona, Bertozzi Stefano M: High risk of HIV in non-brothel based female sex workers in India. BMC Public Health 2005, 5:87-96.

2. Ministry of Health of China, UNAIDS, WHO: 2009 Update on the HIV/AIDS Epidemic and Response in China. Beijing:Ministryof Health; 2009.

3. China National Bureau of Statistics: Characteristics of Chinese rural migrants. Beijing: China National Bureau of Statistics; 2002. 2000

4. Anderson AF, Qingsi Z, Hua X, Jianfeng B: China's floating population and the potential for HIV transmission: a socialbehavioural perspective. AIDS Care 2003, 15:177-185.

5. Hu Z, Liu H, Li X, Stanton B, Chen X: HIV-related sexual behaviour among migrants and non-migrants in a rural area of China: role of rural-tourban migration. Public Health 2006, 120:339-345.

6. He N, Detels R, Chen Z, Jiang Q, Zhu J, Dai Y, Wu M, Zhong X, Fu C, Gui D: Sexual behavior among employed male ruralmigrants in Shangai, China. AIDS Educ Prev 2006, 18:176-186.

7. Huang Yingying, Hederson Gaile, Pan Suiming, Cohen Myrons: HIV/AIDS risk among brothel-based female sex workers in China: Assessing the terms, content, and knowledge of sex work. Sexually Transmitted Diseases. 2004, 31(11):695-700 
8. Shan GN: Zhongguo Changji: Guoqu yu Xianzai (Prostitutes in China:The Past and Present). Beijing: Falu Chubanshe Press; 1995.

9. Hershatter G: Dangerous Pleasure. Prostitution and Modernity in Twentieth-Century Shanghai. Berkeley: University of California Press; 1997.

10. Roua Keming, Wua Zunyou, Sullivana Sheena G: A five-city trial of a behavioural intervention to reduce sexually transmitted disease/HIV risk among sex workers in China. AIDS 2007, 21(suppl 8):S95-S101.

11. Eldridge Sandra M, Ukoumunne Obioha C, Carlin John B: The IntraCluster Correlation Coefficient in Cluster Randomized Trials: A Review of Definitions. International Statistical Review 2009, 77(3):378-394.

12. Feldblum PJ, Kuyoh M, Omari M, Ryan KA, Bwayo JJ, Welsh M: Baseline STD prevalence in a community intervention trial of the female condom in Kenya. Sex Transm Inf 2000, 76:454-456.

13. Feldblum Paul J, Kuyoh Maureen A, Bwayo Job J, Omari Mohamed, Wong Emelita L, Tweedy Kathryn G, Welsh Michael J: Female condom introduction and sexually transmitted infection prevalence: results of a community intervention trial in Kenya. AIDS 2001, 15(8):1037-1044.

14. Rao JNK, Scott AJ: A simple method for the analysis of clustered binary data. Biometrics 1992, 48:577-585

15. Tucker Joseph D, Henderson Gail E, Wang Tian F: Surplus men, sex work, and the spread of HIV in China. AIDS 2005, 19:539-547.

16. Reza-Paul Sushena, Beattie Tara, Sye Hafeez Ur Rahman: Declines in risk behaviour and sexually transmitted infection prevalence following a community-led HIV preventive intervention among female sex workers in Mysore, India. AIDS 2008, 22(suppl 5):S91-S100.

17. Rou Keming, Wu Zunyou, Sullivan Sheena G: A five-city trial of a behavioural intervention to reduce sexually transmitted disease/HIV risk among sex workers in China. AIDS 2007, 21(suppl 8):S95-S101.

18. Ding Y, Detels R, Zhao Z, Zhu Y, Zhu G, Zhang B: HIV infection and sexually transmitted diseases in female commercial sex workers in China. J Acquir Immune Defic Syndr 2005, 38:314-319.

19. Lau JT, Tsui HY, Siah PC, Zhang KL: A study on female sex workers in southern China (Shenzhen): HIV-related knowledge, condom use and STD history. AIDS Care 2002, 14:219-233.

20. Qu S, Liu W, Choi KH, Li R, Jiang D, Zhou Y: The potential for rapid sexual transmission of HIV in China: sexually transmitted diseases and condom failure highly prevalent among female sex workers. AIDS Behav 2002, 6:267-275.

\section{Pre-publication history}

The pre-publication history for this paper can be accessed here: http://www.biomedcentral.com/1471-2458/10/377/prepub

\section{doi: 10.1186/1471-2458-10-377}

Cite this article as: Cai et al., A study of HIV/AIDS related knowledge, attitude and behaviors among female sex workers in Shanghai China BMC Public Health 2010, 10:377

Submit your next manuscript to BioMed Centra and take full advantage of:

- Convenient online submission

- Thorough peer review

- No space constraints or color figure charges

- Immediate publication on acceptance

- Inclusion in PubMed, CAS, Scopus and Google Scholar

- Research which is freely available for redistribution

Submit your manuscript at www.biomedcentral.com/submit
C Biomed Central 